\title{
Field-of-view characteristics and resolution matching for the Global Precipitation Measurement (GPM) Microwave Imager (GMI)
}

\author{
Grant W. Petty ${ }^{1}$ and Ralf Bennartz ${ }^{2}$ \\ ${ }^{1}$ Atmospheric and Oceanic Sciences, University of Wisconsin, 1225 W. Dayton St., Madison, WI, 53706, USA \\ ${ }^{2}$ Earth and Environmental Sciences, Vanderbilt University, 5726 Stevenson Center, Nashville, TN 37240, USA \\ Correspondence to: Grant W. Petty (gwpetty@wisc.edu)
}

Received: 23 August 2016 - Discussion started: 31 August 2016

Revised: 19 January 2017 - Accepted: 21 January 2017 - Published: 7 March 2017

\begin{abstract}
Representative parameters of the scan geometry are empirically determined for the Global Precipitation Measurement (GPM) Microwave Imager (GMI). Effective fields of view (EFOVs) are computed for the GMI's 13 channels, taking into account the blurring effect of the measurement interval on the instantaneous fields of view (IFOVs). Using a Backus-Gilbert procedure, coefficients are derived that yield an approximate spatial match between synthetic EFOVs of different channels, using the $18.7 \mathrm{GHz}$ channels as a target and with due consideration of the tradeoff between the quality of the fit and noise amplification and edge effects. Modest improvement in resolution is achieved for the $10.65 \mathrm{GHz}$ channels, albeit with slight "ringing" in the vicinity of coastlines and other sharp brightness temperature gradients. For all other channels, resolution is coarsened to approximate the $18.7 \mathrm{GHz}$ EFOV. It is shown that the resolution matching procedure reduces nonlinear correlations between channels in the presence of coastlines as well as enables the more efficient separation of large brightness temperature variations due to coastlines from the much smaller variations due to other geophysical variables. As a byproduct of this work, we report accurate EFOV resolutions as well as a self-consistent set of parameters for modeling the scan geometry of the GMI.
\end{abstract}

\section{Introduction}

Since the 1970s, satellite passive microwave imagers have played a major role in observing the global environment. Precipitation in particular is an environmental variable that passive microwave sensors are able to observe more re- liably than any other sensor type. In 1997, the Tropical Rainfall Measuring Mission (TRMM), carrying the TRMM Microwave Imager (TMI) among other instruments, was launched as the first satellite specifically designed to measure precipitation (Kummerow et al., 1998). Its successor, the Global Precipitation Measurement (GPM) Core Observatory (Hou et al., 2014), was launched 27 February 2014 and became operational for scientific applications beginning 4 March 2014. In addition to a Dual-frequency Precipitation Radar (DPR), it carries a passive radiometer, the GPM Microwave Imager (GMI; Draper et al. 2015).

The GMI has 13 channels ranging from 10.65 to $183 \mathrm{GHz}$, all but one $(23.80 \mathrm{GHz})$ with dual polarization (Table 1$)$. As is true for most satellite passive microwave radiometers, the angular resolution of each channel is diffraction limited, implying an instantaneous beamwidth - defined by the halfpower $(-3 \mathrm{~dB})$ points on the antenna pattern - proportional to $1 /\left(v D_{\mathrm{A}}\right)$, where $v$ is the channel frequency and $D_{\mathrm{A}}$ is the antenna diameter. The feedhorns for channels at and above $36.64 \mathrm{GHz}$ are under-illuminated so that the actual angular resolution is slightly coarser than that implied by the diffraction limit (J. Munchak, personal communication, 2016). ${ }^{1}$

The instantaneous field of view (IFOV) represents the projection of the angular antenna pattern onto the Earth's surface from the satellite's altitude and with an incidence angle $\theta$ relative to the local normal. Because of practical limits on antenna sizes, microwave radiometers in space invariably have relatively coarse-resolution IFOVs at low frequencies

\footnotetext{
${ }^{1}$ The original frequency specified for channels 6 and 7 was 36.50 GHz. Draper et al. (2015) reported that those channels had been retuned to $36.64 \mathrm{GHz}$ to reduce susceptibility to known sources of interference.
} 
Table 1. Instantaneous (IFOV) and effective (EFOV) fields of view (native) for GMI channels. Channels are identified by their frequency in $\mathrm{GHz}$ and their polarization ( $\mathrm{V}$ is vertical, $\mathrm{H}$ is horizontal).

\begin{tabular}{lrrr}
\hline & \multicolumn{3}{c}{$3 \mathrm{~dB}$ beamwidth (km) } \\
\cline { 2 - 4 } Channel & $\begin{array}{r}\text { Cross-scan } \\
\text { (IFOV) }\end{array}$ & $\begin{array}{r}\text { Along-scan } \\
\text { (EFOV) }\end{array}$ & Along-scan \\
\hline $10.65 \mathrm{~V}, \mathrm{H}$ & 32.1 & 19.4 & 19.8 \\
$18.70 \mathrm{~V}, \mathrm{H}$ & 18.1 & 10.9 & 11.7 \\
$23.80 \mathrm{~V}$ & 16.0 & 9.7 & 10.5 \\
$36.64 \mathrm{~V}, \mathrm{H}$ & 15.6 & 9.4 & 10.3 \\
$89.00 \mathrm{~V}, \mathrm{H}$ & 7.2 & 4.4 & 6.4 \\
$166.00 \mathrm{~V}, \mathrm{H}$ & 6.3 & 4.1 & 5.8 \\
$183.31 \pm 3 \mathrm{~V}$ & 5.8 & 3.8 & 5.6 \\
$183.31 \pm 7 \mathrm{~V}$ & 5.8 & 3.8 & 5.6 \\
\hline
\end{tabular}

(approximately $19 \times 32 \mathrm{~km}$ at $10.65 \mathrm{GHz}$ for the GMI) and progressively higher-resolution IFOVs with increasing frequency (about $4 \times 6 \mathrm{~km}$ at $183 \mathrm{GHz}$ ).

The effective spatial resolution is additionally reduced by the relative motion of the IFOV across the surface during the integration time $\Delta t$ associated with each image pixel, giving rise to the effective field of view (EFOV), which is slightly larger than the IFOV in the direction of that relative motion (Fig. 3). The variable EFOV resolution implies that a pixel centered just off the shore of a landmass could yield an $89 \mathrm{GHz}$ measurement that is completely over ocean while yielding a $10.65 \mathrm{GHz}$ observation that includes nearly equal proportions of land and ocean. In effect, this resolution mismatch between channels and the resulting inconsistency in scene properties introduces a large potential noise source, one that is partially correlated across channels. In particular, proximity to coastlines and other spatial gradients in brightness temperature can degrade the ability of some geophysical retrieval algorithms to produce useful geophysical retrievals unless special care is taken (Bennartz, 1999; McCollum and Ferraro, 2005).

Petty (2013) discussed the issue of separating desired precipitation signatures from unwanted geophysical noise in multichannel microwave observations and presented a dimensional reduction technique to facilitate that separation in the context of Bayesian retrievals. This technique is based on a two-stage principle component decomposition of the multichannel observations, the first of which objectively isolates and normalizes the surface-dependent noise component and the second of which isolates the desired precipitation signature in the form of up to three "pseudochannels" constructed from linear combinations of the TMI's original nine channels. Petty and Li (2013a) demonstrated the dimensional reduction technique applied to resolution-matched data for the TRMM Microwave Imager, and Petty and Li (2013b) showed that the algorithm yielded significant improvements in RMS error over difficult surface types, including coastlines.

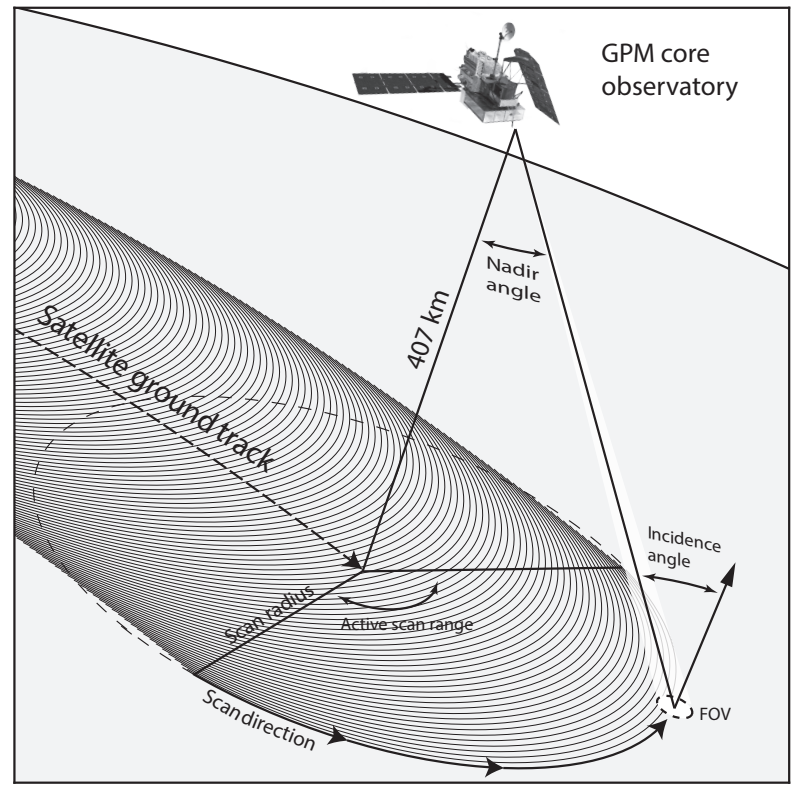

Figure 1. Schematic depiction of the scan geometry of the GMI. The dashed circle represents the instantaneous intersection of the cone of constant incidence angle with the Earth's surface. Its radius and other characteristic parameters are given in Table 2.

To date, similar resolution-matched data have not been available for GMI. In adapting the algorithm of Petty and $\mathrm{Li}$ (2013a) to GMI, the concern arose that the noise associated with unmatched EFOVs would degrade the efficiency with which precipitation signatures could be separated from background variability, especially in the vicinity of coastlines and other sharp brightness temperature gradients. These concerns are the primary motivation for undertaking the work described in this paper.

Our objective here is thus to describe the results of a resolution matching algorithm applied to the nine GMI channels spanning 10.65 through $89 \mathrm{GHz}$. Specifically, we aim to bring all of these channels as close as possible into conformance with the native EFOV of the $18.7 \mathrm{GHz}$ channels.

We do not attempt resolution matching for the highestfrequency channels (166 GHz and higher) because they are separately scanned in a way that does not preserve a fixed geometric relationship with the lower-frequency channels; thus, a fixed set of averaging coefficients is not possible. In principle, however, coefficients could be determined for various fractional offsets between the low-frequency and highfrequency scans. Such a method might give superior results to the nearest-neighbor matching currently utilized in some brightness temperatures products, though no current applications of the high-frequency channels are known to the authors to be sensitive to slight differences in the matching algorithm.

As a byproduct of this work, we tabulate the precise EFOV resolutions for all GMI channels, and we report a concise, self-consistent set of fixed parameters that collectively de- 
scribe, to a reasonable approximation, the observed postlaunch scan geometry of the GMI. While these are not a substitute for the detailed ephemeris and navigation data provided with the imagery for each orbit, they may be useful for the realistic simulation of GMI images from atmospheric and terrestrial models.

\section{Native sensor characteristics}

\subsection{Overview}

The GMI is a conically scanning radiometer whose antenna beam maintains an approximately constant incidence angle with respect to the Earth's surface as it rotates about the vertical axis that connects the satellite with the satellite's subpoint (Fig. 1). The parameters of importance include (a) the relative speed of the satellite subpoint across the Earth's surface, which is determined by the orbital period and, to a far lesser degree, by the Earth's own rotation; (b) the rotation rate of the antenna; (c) the incidence angle of the antenna beam and thus the angular radius of the scan; (d) the integration time $\Delta t$, which determines both the along-scan separation between pixels and the smearing effect that expands the EFOV relative to the IFOV; and (e) the total number of sampled pixels along one scan. The latter is in turn tied to the fraction of one complete circular scan that is actually sampled as well as to the total swath width.

Note that there are two different sets of feedhorns associated with the $10.65-89 \mathrm{GHz}$ channels (1-9) and with the 166 and $183.3 \mathrm{GHz}$ channels (10-13). The latter channels view the Earth at a slightly steeper angle; consequently, their data swath is narrower and their scan pattern is spatially misregistered with that of the lower-frequency channels, as shown in Fig. 2. Finally, because of the oblateness of the Earth, the relative registration in the along-track direction fluctuates by up to several tenths of the spacing between scan lines.

\subsection{Scan geometry model}

The geometry of the GMI scans must be accurately modeled to compute both the actual EFOV sizes and shapes and the overlap between adjacent EFOVs. Both are required in order to be able to determine the correct weights for constructing synthetic (resolution-matched) fields of view (FOVs) for each channel.

Here we model the orbit of the GMI as circular with fixed altitude above the Earth's surface and fixed period, and we ignore the oblateness of the Earth. By carefully examining actual post-launch GMI data, a set of geometrically selfconsistent values for all major parameters of the scan geometry was either directly measured or inferred. These values are reported in Table 2 .

Note that we ignore the variable correction due to the Earth's own rotation, but we introduce a small constant correction to the subtrack velocity relative to that predicted from

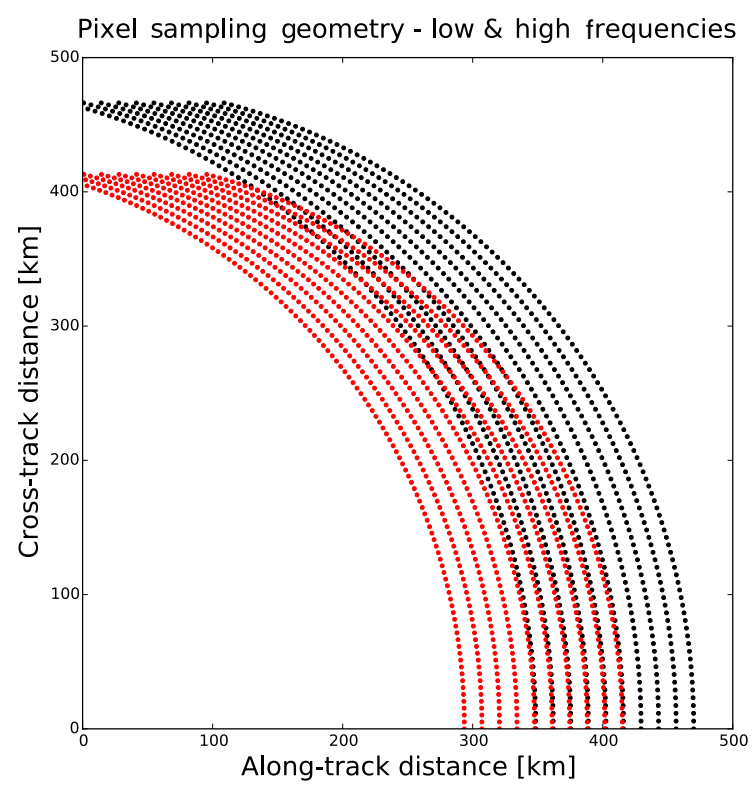

Figure 2. The modeled spatial relationship between pixel centers for $10.65-89$ (black) and 166-183.3 GHz (red). The horizontal axis gives along-track distance from an arbitrary starting point; the vertical axis gives cross-track distance measured from the satellite subtrack.

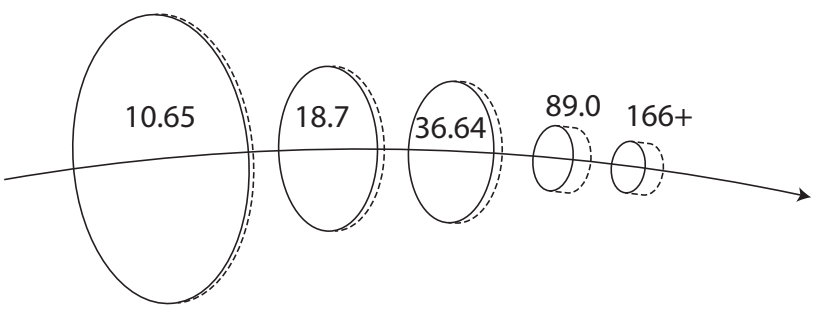

Figure 3. Schematic depiction of the difference between the instantaneous field of view (IFOV; solid lines) and the effective field of view (EFOV; dashed lines) for different channels of the GMI, as measured at the half-power points of the effective antenna functions.

the orbital velocity at the given altitude. Thus, our model can be thought of as approximating the mean scan geometry of the GMI while being subject to minor fluctuating errors that are negligible for nearly contiguous pixels but larger for widely separated pixels.

\subsection{EFOV properties}

The EFOV of each GMI channel results from convolving the IFOV, or the antenna gain pattern projected onto the Earth's surface, with the spatial displacement due to scanning and satellite motion during the integration time. The IFOV is currently modeled as Gaussian, as measured sidelobe gains are at least $30 \mathrm{~dB}$ below that of the main beam for all channels and are negligible for the present purpose. The 
Table 2. Measured and inferred satellite and sensor characteristics determined from actual GMI data so as to construct a self-consistent geometric scan model. These values should be considered typical rather than absolute.

\begin{tabular}{|c|c|c|}
\hline \multicolumn{2}{|l|}{ Parameter } & Value \\
\hline \multicolumn{2}{|c|}{ Altitude } & $407.16 \mathrm{~km}$ \\
\hline \multicolumn{2}{|c|}{ GMI geographic coverage (low freq.) } & to $\pm 69.4^{\circ}$ latitude \\
\hline \multicolumn{2}{|c|}{ Orbital period } & $5554 \mathrm{~s}$ \\
\hline \multicolumn{2}{|l|}{ Scans per orbit } & 2963 \\
\hline \multicolumn{2}{|l|}{ Scan direction } & Counterclockwise \\
\hline \multicolumn{2}{|l|}{ Scan period } & $1.874 \mathrm{~s}$ \\
\hline \multicolumn{2}{|l|}{ Scan range } & $152.6^{\circ}$ \\
\hline \multicolumn{2}{|l|}{ Pixels per scan } & 221 \\
\hline \multicolumn{2}{|l|}{ Integration time } & $3.594 \mathrm{~ms}$ \\
\hline \multicolumn{2}{|c|}{ Along-track scan separation } & $13.15 \mathrm{~km}$ \\
\hline \multicolumn{2}{|c|}{ Scan displacement between low and high frequencies } & $4.1 \pm 0.1$ scans \\
\hline \multirow[t]{2}{*}{ Scan radius (great circle) } & Low freq. & $480.7 \mathrm{~km}$ \\
\hline & High freq. & $426.0 \mathrm{~km}$ \\
\hline \multirow[t]{2}{*}{ Swath width } & Low freq. & $931.2 \mathrm{~km}$ \\
\hline & High freq. & $825.4 \mathrm{~km}$ \\
\hline \multirow[t]{2}{*}{ Along-scan pixel separation } & Low freq. & $5.787 \mathrm{~km}$ \\
\hline & High freq. & $5.130 \mathrm{~km}$ \\
\hline \multirow[t]{2}{*}{ Earth incidence angle } & Low freq. & $52.78^{\circ}$ \\
\hline & High freq. & $49.11^{\circ}$ \\
\hline
\end{tabular}

Table 3. Comparison of native and resolution-matched EFOV $3 \mathrm{~dB}$ widths $(\mathrm{km})$.

\begin{tabular}{ccc|cc}
\hline & \multicolumn{2}{c|}{ Cross-scan } & \multicolumn{2}{c}{ Along-scan } \\
\cline { 2 - 5 } Frequency & Native & Matched & Native & Matched \\
\hline 10.65 & 32.1 & 26.5 & 19.8 & 16.5 \\
18.70 & 18.1 & 18.1 & 11.7 & 11.7 \\
23.80 & 16.0 & 18.0 & 10.5 & 11.7 \\
36.64 & 15.6 & 18.0 & 10.3 & 11.7 \\
89.00 & 7.2 & - & 6.4 & 11.7 \\
\hline
\end{tabular}

beamwidths were determined from the half-power points of field-measured antenna patterns supplied to us by C. Kummerow (personal communication, 2014) and consistent with those employed by Draper et al. (2015). The computed IFOV and EFOV dimensions are reported in Table 1, and a schematic depiction of the change in EFOV resolution relative to the IFOV is shown in Fig. 3. Because the smearing effect of the time integration is almost entirely in the alongscan direction, only that dimension is measurably changed for the EFOV relative to the IFOV. It is most pronounced for the highest-resolution IFOVs.

Note that the interscan distance of $13.15 \mathrm{~km}$ is significantly larger than the cross-scan EFOV dimension of $7.2 \mathrm{~km}$ for the $89.00 \mathrm{GHz}$ channels. In other words, these channels provide noncontiguous coverage. That in turn implies that no spatial average of $89 \mathrm{GHz}$ EFOVs can closely approximate the EFOV of any lower-frequency channel.

\section{FOV-matching methodology}

\subsection{Overview}

To address the large mismatch in EFOV sizes between lowerand higher-frequency channels, we have two choices. We can

- spatially average (convolve) higher-resolution channels to approximately match the coarser resolution of a lower-frequency channel or

- sharpen (deconvolve) the lowest-resolution channel(s) to approximate a higher-frequency channel's EFOV.

In both cases, resolution matching requires one to linearly combine the observations from a set of contiguous pixels so as to approximate the desired target EFOV. The new (synthetic) EFOV is simply the weighted sum of the original EFOVs. To achieve resolution sharpening, there must be both positive and negative weights, but they must all sum to unity to conserve the total radiance in the image.

It must be emphasized that it is generally not possible to achieve a perfect match. One can only aim to achieve the best possible match and then examine the empirical quality of the outcome. This is especially true in the case of deconvolution, as weighting coefficients must be determined so as to achieve 
reasonable improvements in resolution without unwanted artifacts such as excessive noise amplification and/or "ringing". Also, deconvolution is only possible when the pixel spacing is significantly smaller than the size of the EFOV whose resolution one is seeking to improve. As a practical matter, this limits the use of deconvolution for the GMI to the $10.65 \mathrm{GHz}$ channels. Finally, the ability to match FOVs is degraded at the edge of the swath, where there is an incomplete set of overlapping or contiguous pixels.

Our efforts here are similar to those reported for earlier microwave imagers, such as the Special Sensor Microwave Imager (SSM/I) (Farrar and Smith, 1992; Robinson et al., 1992), the TRMM Microwave Imager (Bauer and Bennartz, 1998), the Advanced Microwave Sounding Unit (Bennartz, 2000), and the Advanced Microwave Scanning Radiometer for the Earth Observing System (Wang et al., 2011). Apart from Bennartz (2000) and Wang et al. (2011), most of these do not examine the actual properties of the resulting synthetic EFOVs.

\subsection{Coefficient determination}

In the classic method of Backus and Gilbert (1968, 1970), which was in turn adapted to satellite passive microwave imagers by Stogryn (1978), a cost function is defined that incorporates both a measure of noise amplification and a quadratic measure of resolution or "spread". A tuning parameter $\gamma$ allows the relative emphasis on each of the two terms to be varied. The method employed here is essentially the BackusGilbert method, but we replace the second term ("spread") with one representing spatial correlation between the synthetic FOV (constructed from a linear sum of overlapping real EFOVs) and the target EFOV, in this case the native EFOV of the $18.7 \mathrm{GHz}$ channels. Thus, for the $10.65 \mathrm{GHz}$ channels, our procedure attempts to sharpen the resolution within the limits of spatial sampling and noise amplification considerations. For the higher-frequency channels, the procedure leads to a spatial averaging.

For convenient reference, the full mathematical derivation is provided in Appendix A. Farrar and Smith (1992) take a similar approach to ours in their deconvolution of SSM/I brightness temperatures, except that their target EFOV was an idealized uniform disk with sharp edges rather than a real (and therefore smooth) EFOV; otherwise the mathematics is the same.

Fundamentally, the method entails taking a linear sum of multiple FOVs overlapping the target FOV. That is, if the target FOV is denoted $F_{0}(x, y)$, then our goal is to create a synthetic FOV $F^{\prime}(x, y)$ for another channel such that

$F_{0}(x, y) \approx F^{\prime}(x, y)=\sum_{i} w_{i} f_{i}(x, y)$,

where $w_{i}$ is the appropriately chosen linear weight applied to each of the original FOVs (or pixels) $f_{i}$ in the neighborhood of $F_{0}$. Note that to conserve brightness temperature, the weights must sum to unity:

$\sum_{i} w_{i}=1$

The quality of $F^{\prime}$ as an approximation to $F_{0}$ can be defined in various ways. Here, we choose the squared deviation integrated over area:

$\chi^{2}=\iint\left[F^{\prime}(x, y)-F_{0}(x, y)\right]^{2} \mathrm{~d} x \mathrm{~d} y$.

If the channel being operated on has a higher frequency than the reference channel, then its native resolution is generally higher than that of the reference channel. FOV matching then reduces to a spatial averaging or blurring procedure, and most or all of the coefficients in Eq. (1) are positive. If, however, coarser-resolution FOVs are being combined in an effort to match a finer-resolution target FOV, then this amounts to a deconvolution, or sharpening procedure, and the weights will necessarily be both positive and negative as needed to cancel the response outside the target FOV.

A well-known problem with deconvolution, when not done carefully, is that the individual magnitudes of $w_{i}$ can become quite large (while still satisfying Eq. 2), leading to severe noise amplification as well as "ringing" in the deconvolved image in the presence of sharp brightness temperature gradients. The measure of the noise variance amplification associated with a linear filter is

$N^{2}=\sum_{i} w_{i}^{2}$,

since the effective noise variance in the processed image is then

$\sigma_{\text {post }}^{2}=N^{2} \sigma_{\text {pre }}^{2}$,

where $\sigma_{\text {pre }}^{2}$ is the "native" noise present in the original image, including possibly geophysical noise and/or uncertainties in the precise FOV shape in addition to instrument noise.

Given Eq. (2), $N^{2}$ is absolutely minimized when the $w_{i}$ are all positive and equal, corresponding to a pure averaging or blurring procedure. In contrast, $N^{2}$ can become arbitrarily large when pushing the limits of a deconvolution or sharpening procedure. In any case, whether sharpening or blurring the image, it is important to consider the inevitable tradeoff between achieving the best possible fit to the target FOV and controlling noise amplification and ringing.

Even apart from noise amplification considerations, it is generally impossible to exactly match an arbitrary target FOV via a sum over the discrete set of neighboring FOVs of different size and shape. This is especially true when the pixel density (spatial sampling) is poor relative to the resolution of the target FOV. The target FOV is therefore indeed only a target and is never actually achieved in the footprint matching procedure. Rather, one must examine the resulting synthetic FOV $F^{\prime}$ to determine how good the fit actually is and whether the procedure is of sufficient utility to be worth the effort. 
(a) $10.65 \mathrm{GHz}$
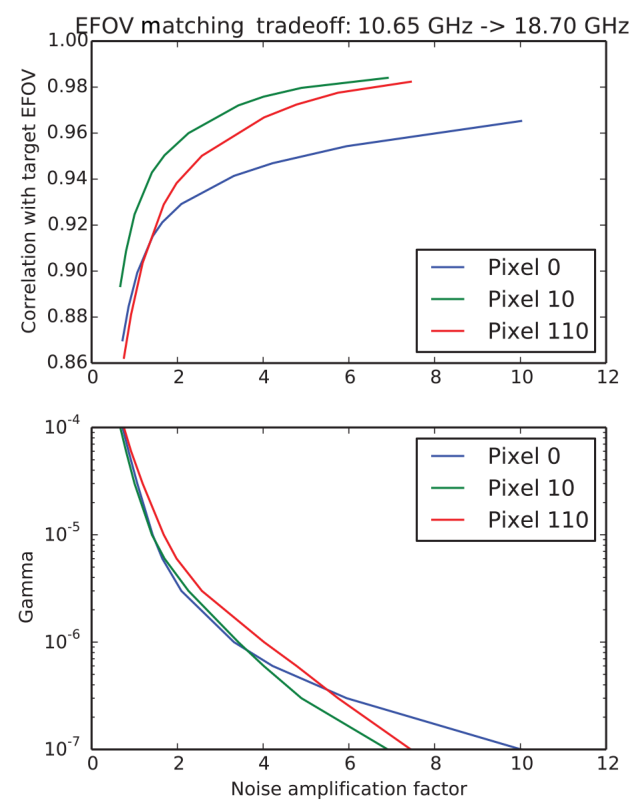

(c) $36.64 \mathrm{GHz}$
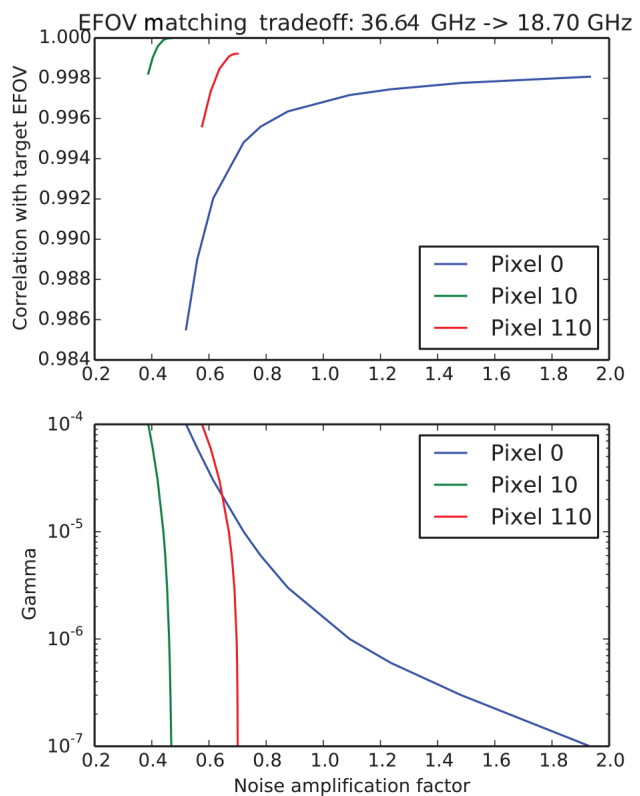

\section{(b) $23.80 \mathrm{GHz}$}
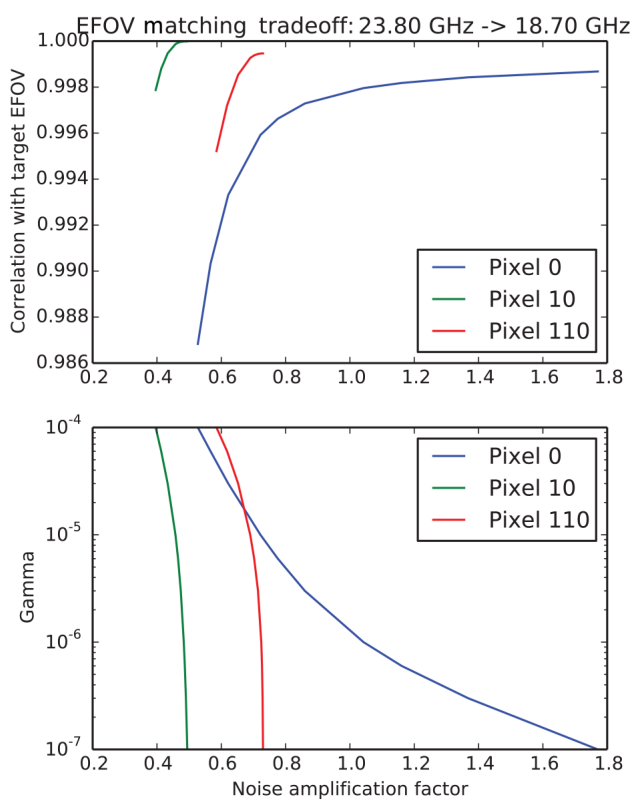

(d) $89.0 \mathrm{GHz}$
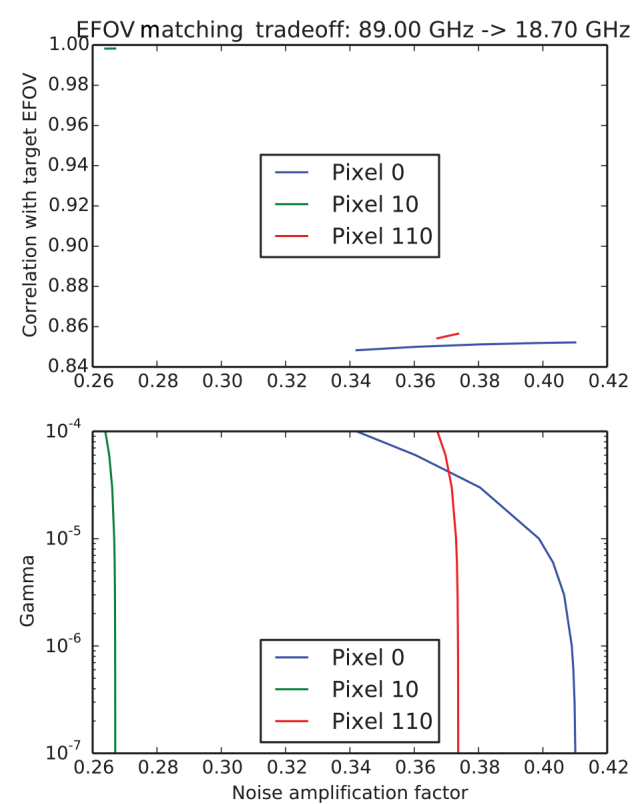

Figure 4. Relationship between noise factor, fit to the target EFOV, and the gamma parameter for the (a) 10.65, (b) 23.80, (c) 36.64, and (d) $89.0 \mathrm{GHz}$ channels. For each frequency, the top panel depicts the tradeoff between the fit (as indicated by the spatial correlation coefficient) and the noise factor; the bottom panel depicts the relationship between the gamma parameter and the noise factor. Pixel 0 is the first pixel in the scan, where the possibility for EFOV matching is partially limited by the absence of overlapping pixels beyond the edge of the swath. Pixel 10 is an interior pixel with comparatively high spatial sampling density, allowing for the best fit. Pixel 110 is at the center of the swath, where the sampling density is lowest.

\subsection{Noise vs. fit}

Figure 4 depicts the tradeoffs between noise factor and fit to the target EFOV. For $10.65 \mathrm{GHz}$, we did not want to exceed a noise amplification factor of about 2 , which limited the qual- ity of the fit to the target EFOV defined by the $18.70 \mathrm{GHz}$ channels. Even without this constraint, the fit could only be improved by a few percent. For 23.80 and $36.64 \mathrm{GHz}$, an excellent fit approaching $100 \%$ is achievable for all but the edge pixels without any noise amplification. For 89.00 , a rel- 
(a) Center EFOVs for $10.65 \mathrm{GHz}$ (cross-scan)
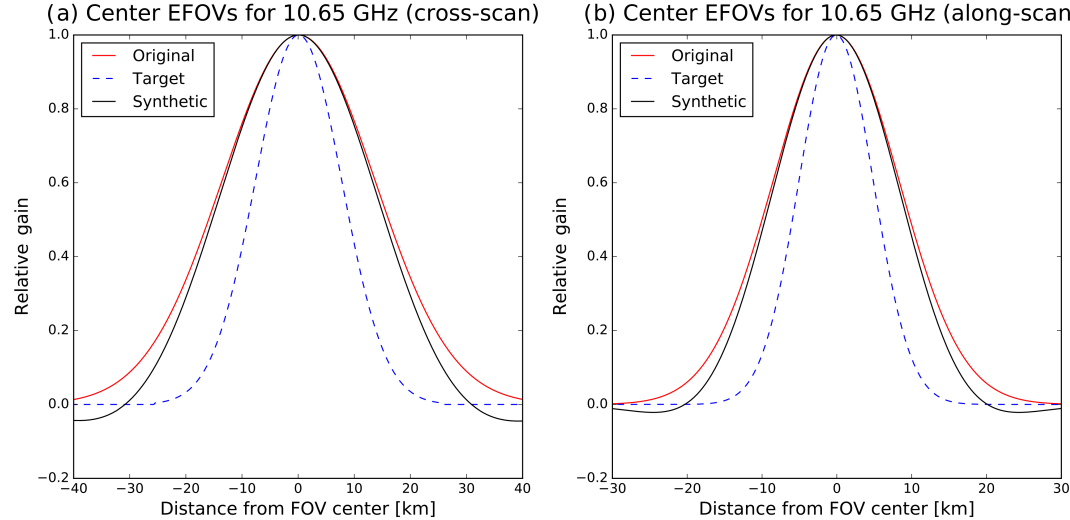

(c) Center EFOVs for $23.80 \mathrm{GHz}$ (cross-scan)
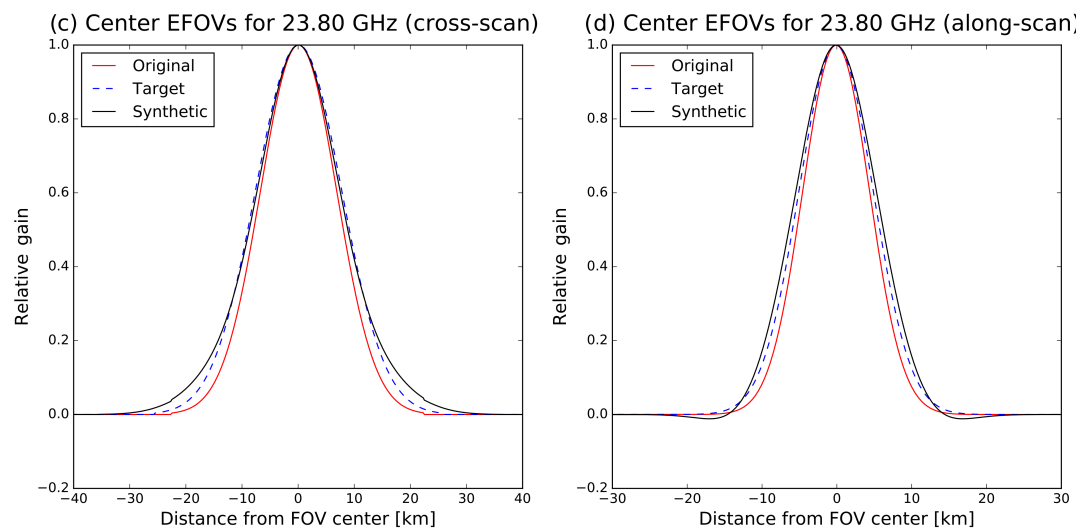

(e) Center EFOVs for $36.64 \mathrm{GHz}$ (cross-scan)
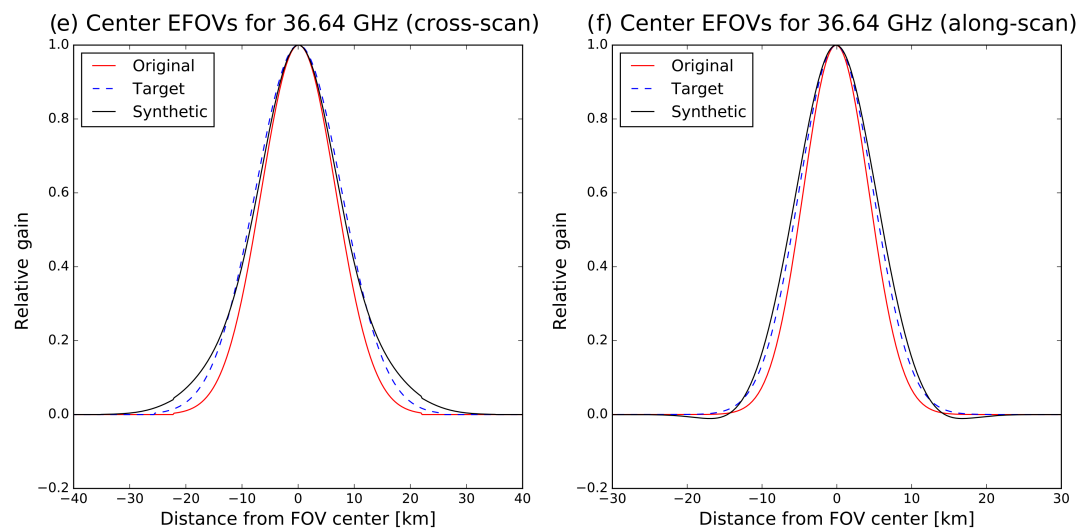

(g) Center EFOVs for $89.00 \mathrm{GHz}$ (cross-scan)
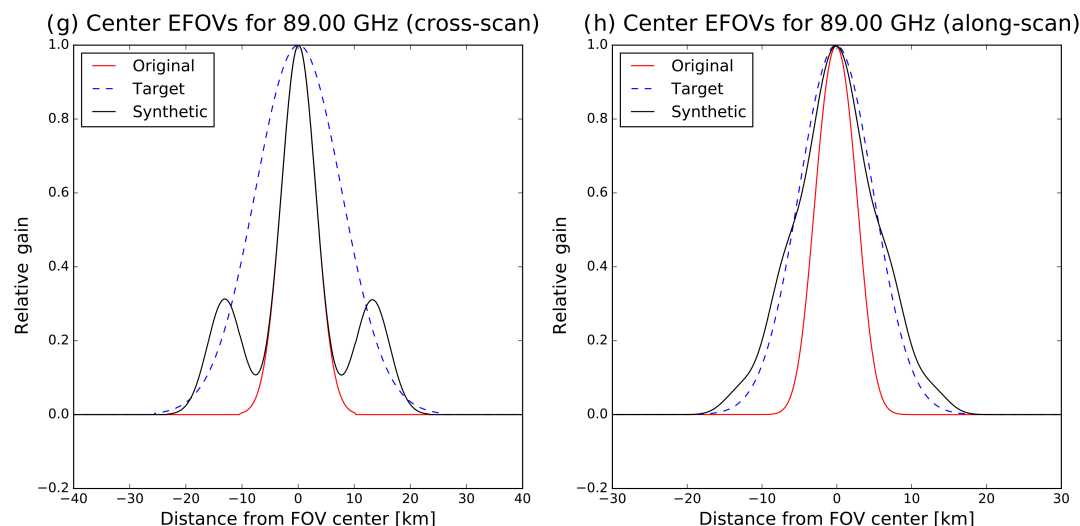

Figure 5. From top to bottom, the shape of the final synthetic EFOVs at the center of the swath for 10.65, 23.80, 36.64, and 89.0 GHz (solid curves). For comparison, the native EFOVs (red curve) and target $18.70 \mathrm{GHz}$ EFOVs (blue dashed curve) are shown. The left column is for the cross-scan direction; the right column is for the along-scan direction. 

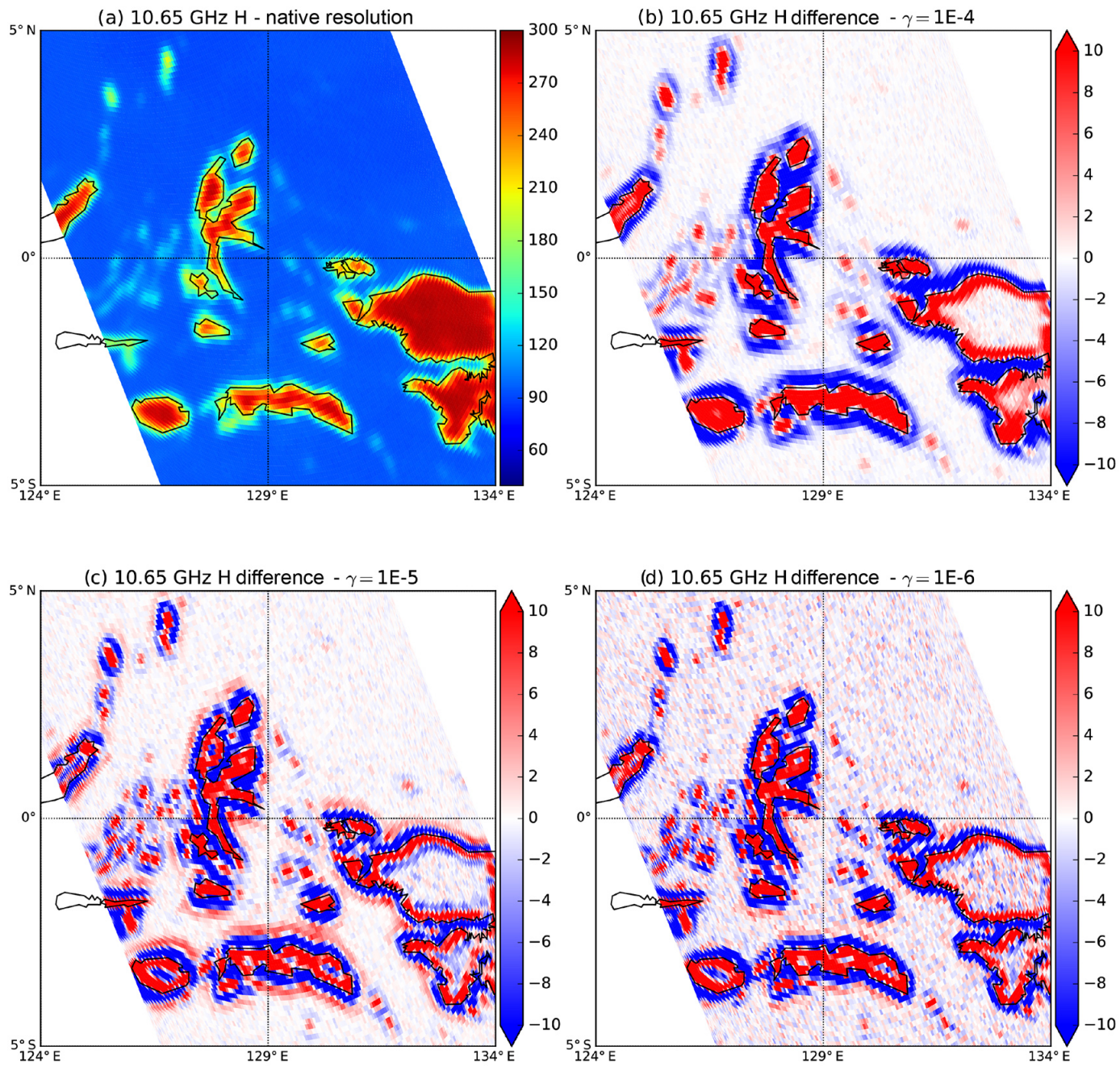

Figure 6. The effect of variations of the tuning parameter $\gamma$ on the deconvolution of the $10.65 \mathrm{GHz}$ channels (brightness temperatures in $\mathrm{K}$ ) as applied to real data. (a) Native resolution brightness temperatures [K]. (b) Differences between native and adjusted resolution for $\gamma=1.0 \times 10^{-4}$. (c) and (d) same as (b) but $\gamma=1.0 \times 10^{-5}$ and $\gamma=1.0 \times 10^{-6}$, respectively.

atively poor fit is achieved due to significant undersampling by the native FOVs in the cross-scan direction. Overall, we find that a constant tuning value of $\gamma=6 \times 10^{-6}$ yields a reasonable compromise between fit and noise amplification for all channels. The resulting coefficients are available for download from Petty and Bennartz (2016).

\section{Results}

\subsection{Synthetic EFOVs}

Figure 5 depicts the shapes of the final synthetic EFOVs for pixel 110 (center of the swath). Of particular note are the following points. While there is slight improvement in the $10.65 \mathrm{GHz}$ fit to the target $18.70 \mathrm{GHz} \mathrm{EFOV}$, it is not possible to actually match that resolution. As found previously by Bauer and Bennartz (1998) for the TRMM Mi- crowave Imager, the improvement is somewhat better in the along-scan direction due to more oversampling in that direction. There are significant negative sidelobes in the synthetic EFOVs for $10.65 \mathrm{GHz}$. This appears to be unavoidable given the available sampling for these channels. The fit for 23.80 and $36.64 \mathrm{GHz}$, however, is excellent.

Because the $89.00 \mathrm{GHz}$ channels are badly undersampled in the cross-scan direction, the synthetic FOV fit to the target EFOV is poor in that direction. It is quite good in the alongscan direction. All of the above results are the worst cases for the entire interior of the data swath, as the sampling density improves toward the edges. At the edges, however, the fit deteriorate again. Table 3 gives the half-power beamwidths of the synthetic EFOVs compared to the native resolution for each channel. (Note that the half-power value does not give a useful measure of the improvement in the fit for $89.00 \mathrm{GHz}$ due to the multimodal shape.) 
(a) $10.65 \mathrm{GHz} \mathrm{H}$ - native resolution

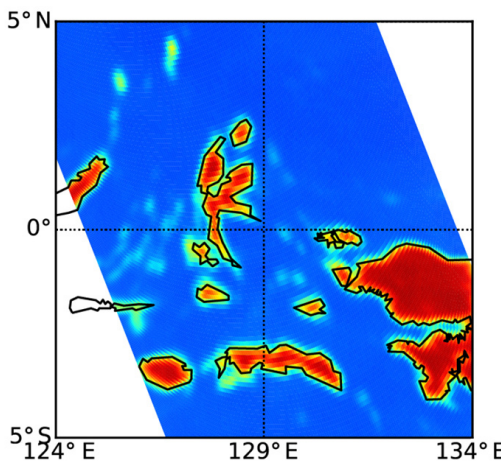

(c) $18.7 \mathrm{GHz} \mathrm{H}$ - native resolution

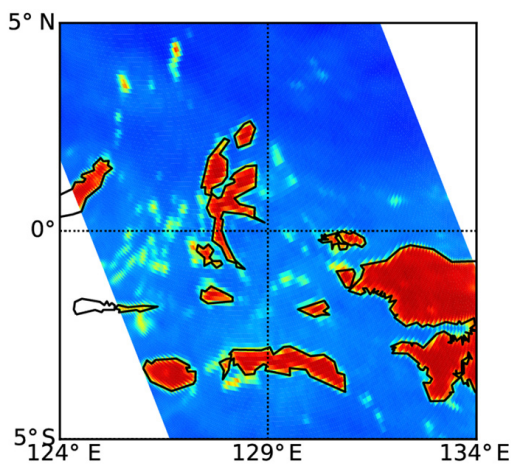

(e) $36.64 \mathrm{GHz} \mathrm{H}$ - native resolution

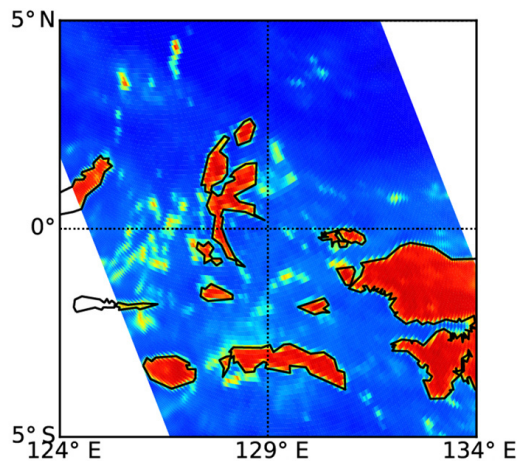

(g) $89.0 \mathrm{GHz} \mathrm{H}$ - native resolution

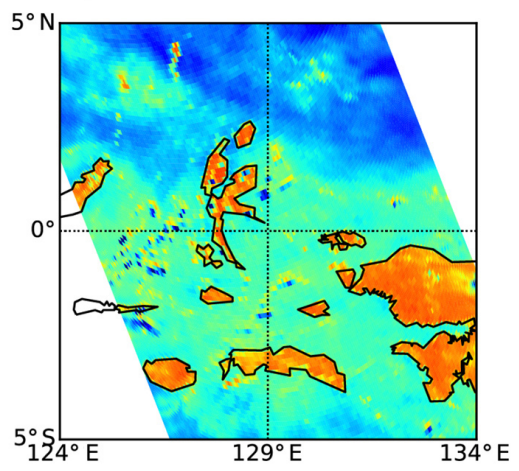

(b) $10.65 \mathrm{GHz} \mathrm{H}$ - matched resolution

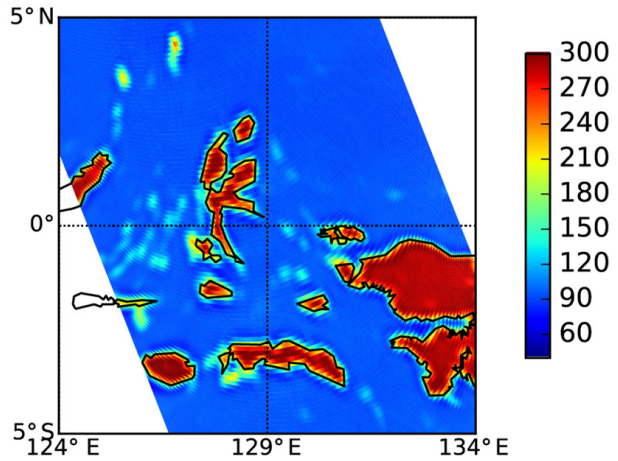

(d) $18.7 \mathrm{GHz} \mathrm{H}$ - matched resolution

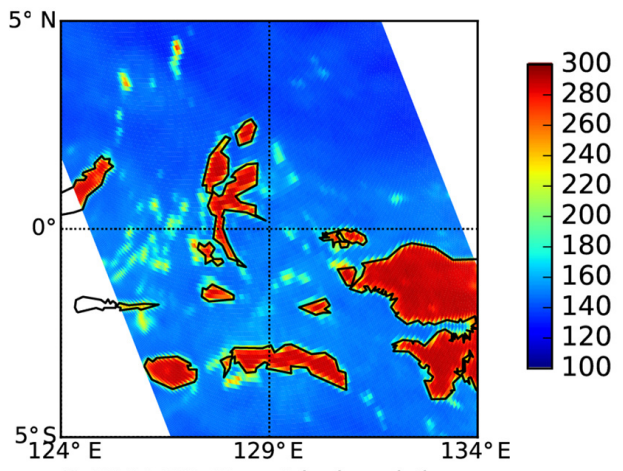

(f) $36.64 \mathrm{GHz} \mathrm{H}$ - matched resolution

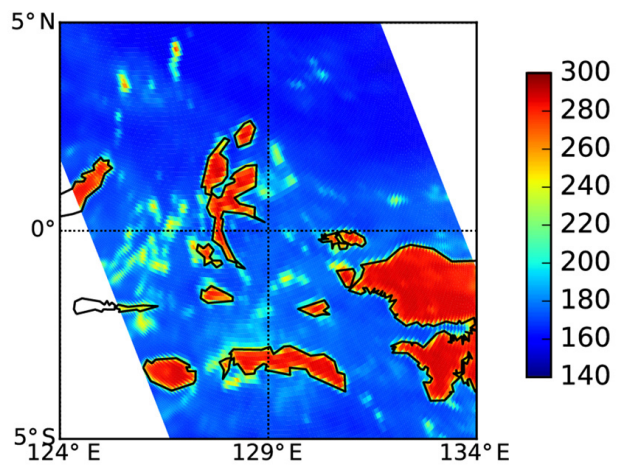

(h) $89.0 \mathrm{GHz} \mathrm{H}$ - matched resolution

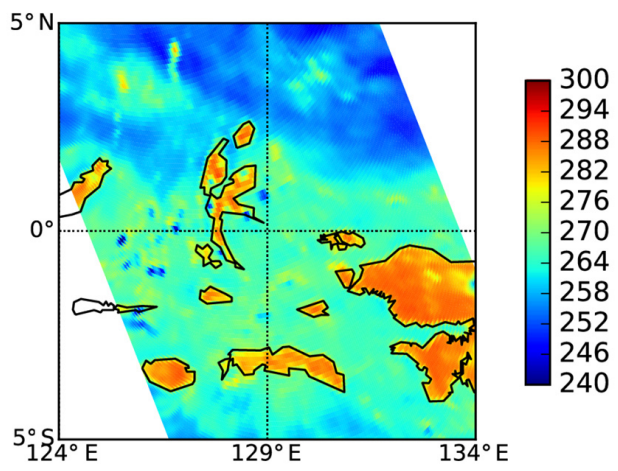

Figure 7. A sample of GMI imagery before (left column) and after (right column) the resolution matching procedure. For $18.70 \mathrm{GHz}$, which defines the target EFOV, no adjustment is made. Results are not shown for $23.8 \mathrm{GHz}$, as the effects of the resolution matching are almost imperceptible at this frequency. 

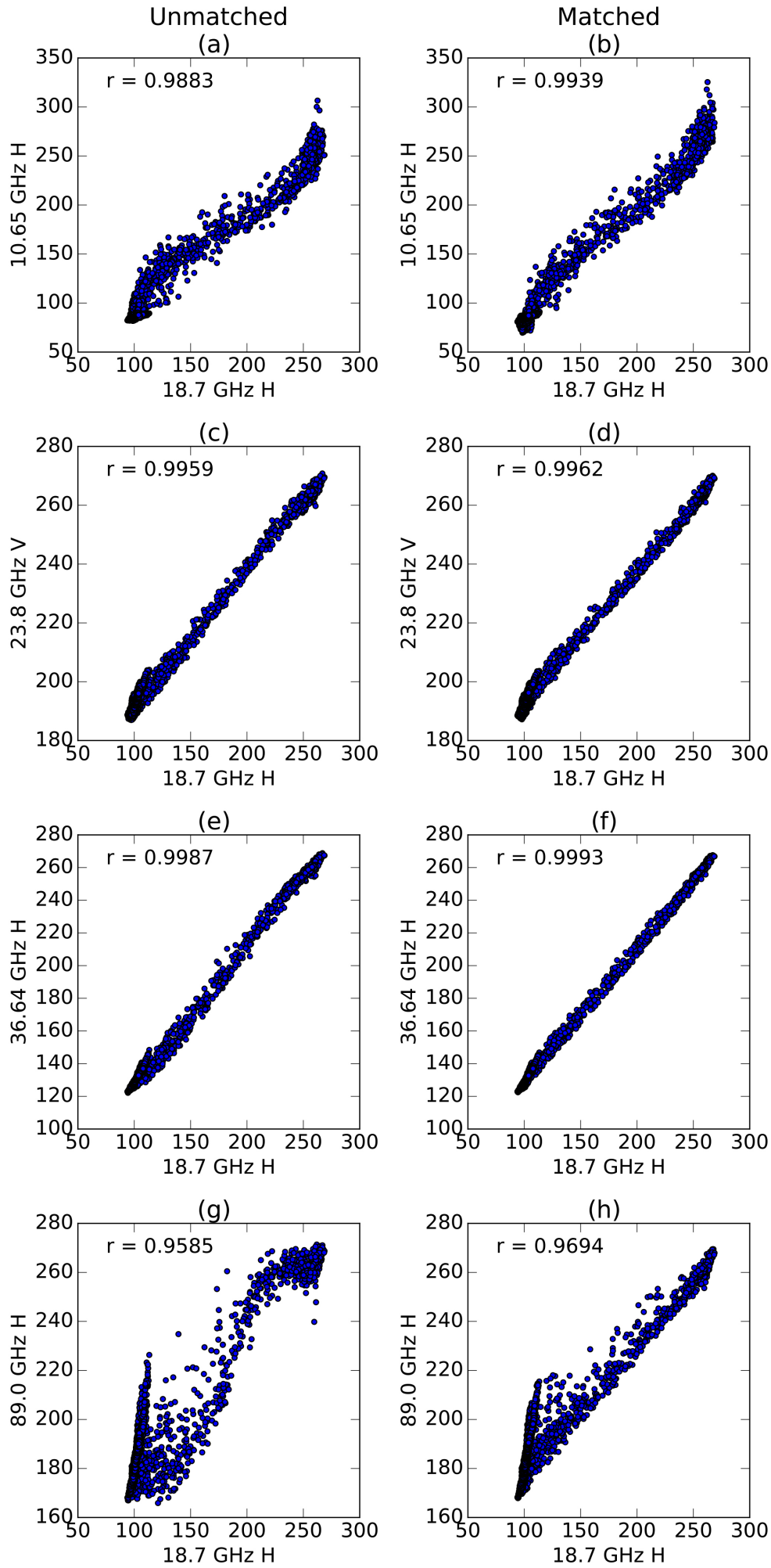

Figure 8. Scatter plots of selected GMI channels against the co-located $18.7 \mathrm{GHz}$ horizontal channel brightness temperatures (K) for a swath segment over coastal areas of Italy and Greece on 13 December 2015 (orbit number 10167). These plots demonstrate the improved linear correlation, in the presence of the sharp spatial gradient in brightness temperature at the coastline, between channels of disparate native spatial resolutions before (left column) and after (right column) the resolution matching procedure is applied. (a, b) $10.65 \mathrm{GHz}$ horizontal; (c, d) $23.8 \mathrm{GHz}$ vertical; (e, f) $36.64 \mathrm{GHz}$ horizontal; (g, h) $89.0 \mathrm{GHz}$ horizontal. 


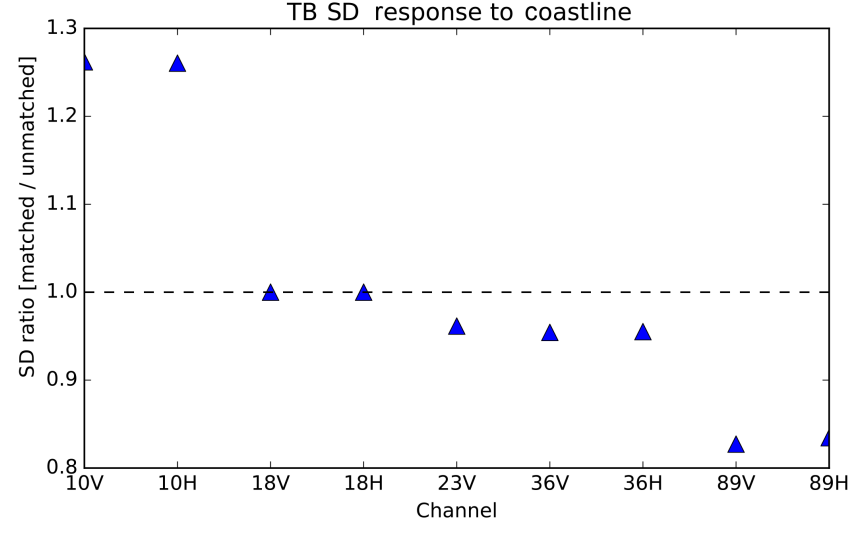

Figure 9. For the coastal data depicted in Fig. 8, the effect of resolution matching on the standard deviations of the brightness temperatures from each channel is shown.

\subsection{Application to real data}

\subsubsection{Visual depiction}

Figure 6 depicts the implications of various values of $\gamma$ for the deconvolution of actual $10.65 \mathrm{GHz}$ imagery. To make the differences most visible, a swath segment was chosen that includes numerous islands as well as some cellular convection. As one progresses to greater sharpening, "overshoot" (Gibbs effect) becomes evident in the vicinity of sharp gradients. Based on our analysis, this appears to be an unavoidable artifact of any significant sharpening of the $10.65 \mathrm{GHz}$ resolution, given the less-than-ideal spatial sampling.

For the chosen value of $\gamma=6 \times 10^{-6}$, Fig. 7 depicts a sample of real GMI data with (right column) and without (left column) the (de)convolution procedure applied. Improved consistency in apparent resolution between channels is apparent in the right column, as expected.

\subsubsection{Correlation improvement}

A quantitative empirical evaluation of the improved resolution consistency between channels can be obtained by plotting the brightness temperature for a particular channels against the channel whose EFOV is the target, in this case the $18.7 \mathrm{GHz}$ channels. We select for scenes in which the dominant brightness temperature variability for all channels is the sharp discontinuity in surface emissivity between ocean and land. When a pair of channels have the same effective spatial resolution, the transition from ocean to land occurs at the same rate for both and one expects a linear relationship with relatively low scatter. When spatial resolutions are different, the lower-resolution channel experiences a longer transition from pure ocean to pure land, so the resulting scatter plot is distinctly $S$ shaped.

Figure 8 shows results for a single overpass over the central Mediterranean, including Italy and parts of Greece. For this subjectively chosen test case, atmospheric water vapor and cloud water content were low so as to reduce unrelated scatter due to different channels' sensitivities to these factors. In each of these plots, the cold endpoint in the lower left corner represents pure ocean for both channels; the warm endpoint in the upper right represents pure land. Between those endpoints are found various combinations of land and ocean. The left column depicts the relationships prior to resolution matching; the right column shows results after all channels have been partially deconvolved or convolved to the target $18.7 \mathrm{GHz}$ resolution.

In all cases, the linear correlation coefficient improves as a consequence of the resolution matching. In the particular case of 10.65 and $89.0 \mathrm{GHz}$ channels, whose native resolutions are farthest from the target value, the resolution matching reduces or eliminates the $S$ shaped curve in the scatter plot, confirming that both channels have been brought into closer congruence with the target EFOV.

\subsection{Implications for precipitation retrievals}

It is not possible to precisely characterize the impact of resolution matching on retrieval errors, as any improvements will be highly context and algorithm specific. Nevertheless, we can, as just one example, examine the efficiency with which coastal signatures can potentially be separated from other geophysical signatures.

From the data depicted in Fig. 8, we computed the $9 \times 9$ channel brightness temperatures covariances for both the unmatched and the matched data. We find that the brightness temperature variances for the $10.65 \mathrm{GHz}$ channels increase following the resolution matching procedure, while those for $23.80 \mathrm{GHz}$ and higher frequencies decrease as a result of the same procedure (Fig. 9). These outcomes are consistent with moderate spatial sharpening (and associated noise amplification) for the $10.65 \mathrm{GHz}$ channels and spatial averaging for the higher-frequency channels.

The algorithm of Petty and Li (2013b) utilizes only the seven channels from 18.7 to $89 \mathrm{GHz}$ in over-land precipitation retrievals. We therefore extracted the covariance matrices (pre- and post-matched) for just those channels and calculated the eigenvectors (principle components) and eigenvalues. By far, the dominant eigenvector for this data set describes the brightness temperature contrast between land and ocean. In the pre-matched data, this eigenvector explains $99.1 \%$ of the total channel variance, leaving $0.9 \%$ unexplained. This unexplained residue necessarily includes all exploitable geophysical signatures (e.g., precipitation) was well as the nonlinear component of the correlations between channels due to the disparate resolutions (see for example Fig. $8 \mathrm{~g}$ ). In the post-match covariances, only $0.4 \%$ of the variance is unexplained by the first eigenvector, implying at least a factor of 2 improvement in the signal-to-noise ratio, and possibly much more. 


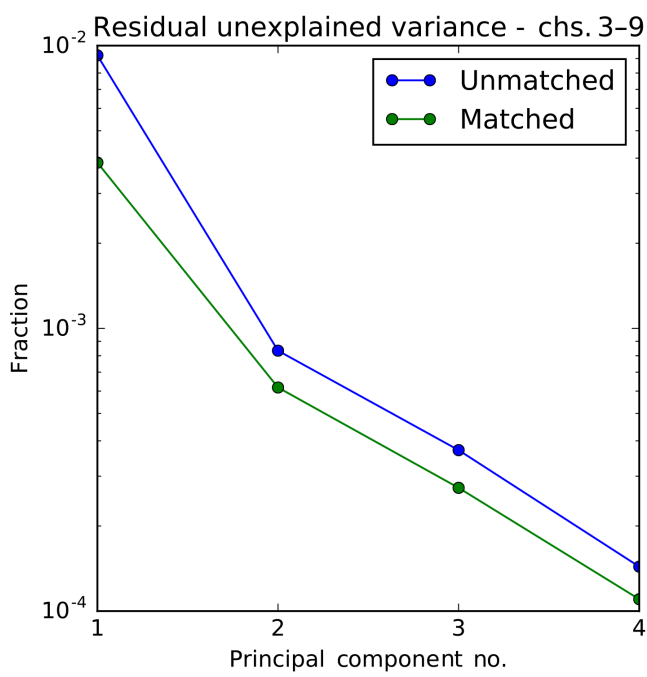

Figure 10. The residual unexplained variance after accounting for the first through fourth principal components (eigenvectors) of the covariance of the 18.7 through $89.0 \mathrm{GHz}$ channels for the coastal data depicted in Fig. 8.

Figure 10 shows how the residual unexplained variance decreases with the inclusion of each successive principle component. We see that a significant reduction is achieved for all of the first four principle components. For reference, the algorithm of Petty and Li (2013a) relies on the first three principle components after variability associated with the background (including coastlines) has been accounted for. We therefore conclude that the resolution matching procedure is likely to improve the ability of the algorithm to distinguish precipitation signatures in the presence of coastlines and other strong gradients in emissivity.

\section{Conclusions}

This paper documents the effective fields of view of the GMI after allowing for the blurring effect of the measurement interval on the instantaneous fields of view. We derived coefficients that produce an approximate spatial match between synthetic EFOVs of different channels using the $18.7 \mathrm{GHz}$ channels as a target and with reasonable tradeoffs between the quality of the fit and noise amplification.
No set of coefficients is capable of generating an ideal match between the $10.65 \mathrm{GHz}$ channels and the target EFOV, because they are not sufficiently densely sampled. There is slight improvement in resolution, but with some edge artifacts in the vicinity of coastlines and other sharp brightness temperature gradients. Depending on the application, one must decide whether the introduced artifacts or the improved resolution are of greater importance.

At $89 \mathrm{GHz}$, the averaging to coarser resolution does not yield a good fit to the $18.7 \mathrm{GHz}$ EFOV because the spacing between $89 \mathrm{GHz}$ scans is too large relative to the cross-scan pixel resolution. Nevertheless, the average is still a significantly better match to the $18.7 \mathrm{GHz}$ EFOV than the unconvolved imagery. For all other channels, the matching procedure yields an excellent fit.

Resolution-matched brightness temperatures based on the coefficients derived herein are currently being utilized in the adaptation of the algorithm of Petty and Li (2013a) to GMI, and we invite other GMI algorithm developers to utilize them as well.

Data availability. Files containing the coefficients are available for electronic download from Petty and Bennartz (2016). 


\section{Appendix A: Derivation}

Our goal is to find the set of weights $w_{i}$ satisfying Eq. (2) that also minimize the cost function

$\Phi=\gamma N^{2}+\chi^{2}$,

where $\gamma$ is a tunable parameter that controls relative importance of noise amplification vs. goodness of fit. Expanding, we have

$$
\begin{aligned}
\Phi & =\gamma \sum_{i} w_{i}^{2} \\
& +\iint\left[\sum_{i} w_{i} f_{i}(x, y)-F_{0}(x, y)\right]^{2} \mathrm{~d} x \mathrm{~d} y .
\end{aligned}
$$

For notational simplicity, the explicit dependence of $f_{i}$ and $F_{0}$ on $(x, y)$ are suppressed in the equations that follow.

Expanding the squared term and taking constant terms outside of the integrals yields

$$
\begin{aligned}
\Phi & =\gamma \sum_{i} w_{i}^{2}+\sum_{i} w_{i} \sum_{j} w_{j} \iint f_{i} f_{j} \mathrm{~d} x \mathrm{~d} y \\
& -2 \sum_{i} w_{i} \iint F_{0} f_{i} \mathrm{~d} x \mathrm{~d} y+\iint F_{0}^{2} \mathrm{~d} x \mathrm{~d} y .
\end{aligned}
$$

The integral terms are all constants, and we may make the following notational substitutions:

$$
\begin{aligned}
P_{i j} & \equiv \iint f_{i} f_{j} \mathrm{~d} x \mathrm{~d} y, \\
q_{i} & \equiv \iint F_{0} f_{i} \mathrm{~d} x \mathrm{~d} y, \\
r & \equiv \iint F_{0}^{2} \mathrm{~d} x \mathrm{~d} y .
\end{aligned}
$$

Employing the Einstein convention of implied summation over pairs of like indices, our cost function can be written simply as

$$
\Phi=\gamma w_{i} w_{i}+w_{j} P_{j k} w_{k}-2 w_{m} q_{m}+r .
$$

We wish to find the coefficients $w$ that minimize $\Phi$ subject to the constraint Eq. (2). The conventional method for solving a constrained optimization problem is the method of Lagrange multipliers. We define a new function:

$$
\begin{aligned}
\Lambda & =\gamma w_{i} w_{i}+w_{j} P_{j k} w_{k}-2 w_{m} q_{m}+r+ \\
& \lambda\left(\sum_{n} w_{n}-1\right),
\end{aligned}
$$

where $\lambda$ is the Lagrange multiplier, and the added term it multiplies is zero when the constraint is satisfied. The task is then to solve the set of equations corresponding to the combination of Eq. (2) with the results of

$$
\frac{\partial}{\partial w_{p}} \Lambda=0 \text {. }
$$

Carrying out the above differentiation yields

$$
\begin{aligned}
\frac{\partial \Lambda}{\partial w_{p}} & =\frac{\partial}{\partial w_{p}}\left[\gamma w_{i} w_{i}+w_{j} P_{j k} w_{k}-2 w_{m} q_{m}\right. \\
& \left.+r+\lambda\left(\sum_{n} w_{n}-1\right)\right] \\
& =2 \gamma w_{p}+2 P_{p k} w_{k}-2 q_{p}-\lambda \\
& =0 .
\end{aligned}
$$

Factoring out the 2 and expressing the last two lines above in matrix notation, we have

$\mathbf{B w}=\mathbf{q}+\frac{\lambda}{2} \mathbf{u}$,

where $\mathbf{u}=(1,1, \cdots, 1)^{T}$, and

$B_{i j}=P_{i j}+\gamma \delta_{i j}$

where $\delta_{i j}$ is the Kronecker delta. That is, the matrix $\mathbf{B}$ is just the matrix $\mathbf{P}$ with the tuning parameter $\gamma$ added to each diagonal element.

The solution for the desired coefficients is then

$\mathbf{w}=\mathbf{B}^{-1}\left[\mathbf{q}+\frac{\lambda}{2} \mathbf{u}\right]$.

We still have the undetermined Lagrange multiplier $\lambda$. Its value follows from the constraint Eq. (2):

$$
\begin{aligned}
\sum_{i} w_{i} & =\sum_{i}\left(\mathbf{B}^{-1} \mathbf{q}\right)_{i}+\frac{\lambda}{2} \sum_{i}\left(\mathbf{B}^{-1} \mathbf{u}\right)_{i} \\
& =\mathbf{u}^{T} \mathbf{B}^{-1} \mathbf{q}+\frac{\lambda}{2} \mathbf{u}^{T} \mathbf{B}^{-1} \mathbf{u} \\
& =1,
\end{aligned}
$$

leading to

$\lambda=\frac{2\left(1-\mathbf{u}^{T} \mathbf{B}^{-1} \mathbf{q}\right)}{\mathbf{u}^{T} \mathbf{B}^{-1} \mathbf{u}}$.

Note that the denominator is just the sum over all elements of $\mathbf{B}^{-1}$. 
Competing interests. The authors declare that they have no conflict of interest.

Acknowledgements. This work was supported by NASA grants NNX13AG49G and NNX16AF70G through the Precipitation Measurement Mission. The suggestions of an anonymous referee as well as referee S. J. Munchak led to significant improvements. Gabriel Shaughnessy provided technical assistance.

Edited by: I. Moradi

Reviewed by: S. J. Munchak and one anonymous referee

\section{References}

Backus, G. and Gilbert, F.: The resolving power of gross earth data, Geophys. J. Roy. Astr. S., 16, 169-205, 1968.

Backus, G. and Gilbert, F.: Uniqueness in the inversion of inaccurate gross Earth data, Philos. T. R. Soc. A, 266, 123-192, 1970.

Bauer, P. and Bennartz, R.: Tropical Rainfall Measuring Mission microwave imaging capabilities for the observation of rain clouds, Radio Sci., 33, 335-349, 1998.

Bennartz, R.: On the use of SSM/I measurements in coastal regions, J. Atmos. Ocean. Tech., 16, 417-431, 1999.

Bennartz, R.: Optimal convolution of AMSU-B to AMSU-A, J. Atmos. Ocean. Tech., 17, 1215-1225, 2000.

Draper, D. W., Newell, D. A., Wentz, F. J., Krimchansky, S., and Skofronick-Jackson, G. M.: The Global Precipitation Measurement (GPM) Microwave Iimager (GMI): Instrument overview and early on-orbit performance, IEEE J. Sel. Top. Appl., 8, 34523462, 2015.

Farrar, M. and Smith, E.: Spatial resolution enhancement of terrestrial features using deconvolved SSM/I microwave brightness temperatures, IEEE T. Geosci. Remote, 30, 349-355, 1992.
Hou, A. Y., Kakar, R. K., Neeck, S., Azarbarzin, A. A., Kummerow, C. D., Kojima, M., Oki, R., Nakamura, K., and Iguchi, T.: The Global Precipitation Measurement Mission, B. Am. Meteorol. Soc., 95, 701-722, doi:10.1175/BAMS-D-13-00164.1, 2014.

Kummerow, C., Barnes, W., Kozu, T., Shiue, J., and Simpson, J.: The Tropical Rainfall Measuring Mission (TRMM) sensor package, J. Atmos. Ocean. Tech., 15, 809-817, 1998.

McCollum, J. and Ferraro, R.: Microwave rainfall estimation over coasts, J. Atmos. Ocean. Tech., 22, 497-512, 2005.

Petty, G. and Li, K.: Improved passive microwave precipitation retrievals over land and ocean, 1. Algorithm description, J. Atmos. Ocean. Tech., 30, 2493-2508, doi:10.1175/JTECH-D-1200144.1, 2013a.

Petty, G. and Li, K.: Improved passive microwave precipitation retrievals over land and ocean, 2. Validation and intercomparison, J. Atmos. Ocean. Tech., 30, 2509-2526, doi:10.1175/JTECH-D12-00184.1, 2013b.

Petty, G. W.: Dimensionality reduction in Bayesian estimation algorithms, Atmos. Meas. Tech., 6, 2267-2276, doi:10.5194/amt6-2267-2013, 2013.

Petty, G. W. and Bennartz, R.: Resolution matching coefficients for the Global Precipitation Measurement (GPM) Microwave Imager (GMI), electronic download, doi:10.5281/zenodo.198835, 2016.

Robinson, W., Kummerow, C., and Olson, W.: A technique for enhancing and matching the resolution of microwave measurements from the SSM/I instrument, IEEE T. Geosci. Remote, 30, 419-429, 1992.

Stogryn, A.: Estimates of brightness temperatures from scanning radiometer data, IEEE Trans. Antenn. Propag., AP-26, 720-726, 1978.

Wang, Y.-Q., Shi, J.-C., Jiang, L.-M., Du, J.-Y., and Tian, B.-S.: The development of an algorithm to enhance and match the resolution of satellite measurements from AMSR-E, Science China Earth Sciences, 54, 410-419, 2011. 